\title{
Morphological features of fruits and seeds of Saposhnikovia divaricata (Apiaceae)
}

\author{
Polina Yugrina ${ }^{1}$, Bayarma Urbagarova $^{2}$, and Tatyana Elisafenko ${ }^{1 *}$ \\ ${ }^{1}$ Central Siberian Botanical Garden of the SB RAS, 630090 Novosibirsk, Russia \\ ${ }^{2}$ Banzarov Buryat State University, 670000, Ulan-Ude, Russia
}

\begin{abstract}
The article presents the characteristics of the fruits and seeds of the medicinal plant Saposhnikovia divaricata (Turcz.) Schischk.), obtained from natural habitats and under conditions of introduction. Heterocarpy has been established, which is expressed in the difference in color, the surface of the mericarp, the size of the mericarp and the seed. The size of the embryo differs slightly. The coefficient of variation of signs is from 9 to 22 $\%$, regardless of the place of origin of the fruits.
\end{abstract}

Currently, the preservation of species with a unique composition of biologically active substances is becoming relevant. The introduction of such species makes it possible to preserve them in the conditions of culture, to study biological features and to develop recommendations for their cultivation. One of these species is Saposhnikovia divaricata (Turcz.) Schischk.) A high content of chromones was found in the roots of plants of this species [1], but the possibility of introduction is poorly studied [2]. The purpose of this work is to study the morphological characteristics of fruits and seeds in plants of $S$. divaricate in vivo and in introduction.

$S$. divaricata is the only species in the endemic genus Saposhnikovia Schischk. the Apiaceae Lindl family. It is found in Russia in the south of the Amur Region, the Republic of Buryatia, the Trans-Baikal Territory, the Primorsky Territory, outside of Russia - in Mongolia, China, on the Korean Peninsula. It grows in meadow stony steppes, on the slopes and trails of hills, on terraces, in steppe shrubbery, sometimes on idle field and on roadsides [3]. This is a perennial monocarpic, with a branched caudex, with a main root forming lateral roots. The stems are glabrous, straight, branching from the base. Shoots of subsequent orders are formed in the axils of the leaves. Synfloriscence is a panicle of compound umbel. Umbrellas are numerous, corymbose. The fruits are cremocarp, consisting of two oblong glabrous mericarpes.

The material was fruits from plants grown from plantlet at the exhibition "Rare and endangered plant species of Siberia" of the Central Siberian Botanical Garden (Novosibirsk) in 2020, as well as collected in the Republic of Buryatia (Tarbagatai district, in 2020), mericarps and seeds of each order were studied. The seeds of the main inflorescence, of the 1st order, were not taken into account, because of there were a small number of them (1-2 fruits). The mass of 1000 mericarpes was determined by weighing 100 pieces 10 times, the arithmetic mean was recalculated by 1000 .

\footnotetext{
* Corresponding author: tveli@ngsl.ru
} 
The macromorphology of fruits and seeds was studied using a Carl Zeiss Stereo Discovery V12 light stereomicroscope with a high-resolution color digital camera AxioCam MRc-5 and with the AxioVision 4.8 program for obtaining, processing and analyzing images. Previously, 20 mericarpies were soaked for a day. the color and structure of the surface of the mericarp and seed, the length and width of the mericarp, commissura, seed, embryo, cotyledon, the axis of the embryo, the ratio of these indicators were determined. Statistical processing of the obtained data was carried out according to G. N. Zaitsev (Zaitsev, 1991), determined the arithmetic mean (M), its error (m), the coefficient of variation $(\mathrm{V})$, the Student's criterion with a probability of $95 \%$.

The fruiting of $S$. divaricata is stretched, the fruits of the inflorescence of the 1 st order ripen first, then fruits of the 2 nd order on the branching shoots in the basal part of the main shoot, after that in the umbrellas of the second order in the acropetal part of the main shoot and in the umbrellas of the third order in the basal part of the main shoot. The fruits of $S$. divaricata break up into two equal mericarps, which have a common appearance for all orders (Fig.1).

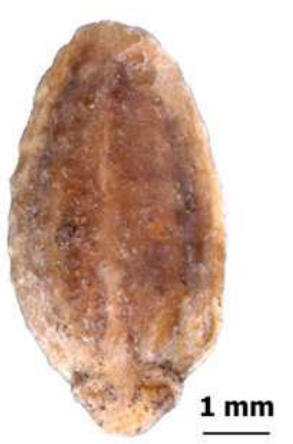

A

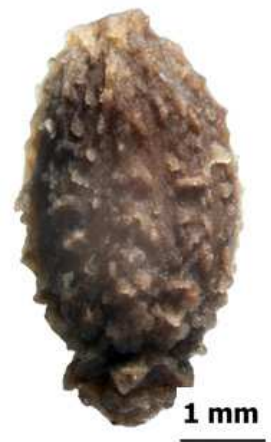

B

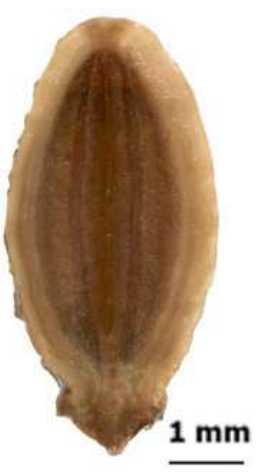

C

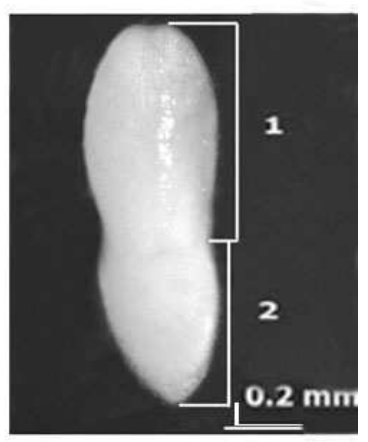

D

Fig. 1. Mericarp and the embryo of Saposhnikovia divaricata. A, B - mericarp, C - commissura mericarp, D - embryo, 1 - cotyledons, 2 - axis of the embryo.

They are different shades of brown in, elliptical, flattened in the dorsoventral direction, more than $6 \mathrm{~mm}$. There are 5 primary edges that do not differ in color. The surface of the exocarp is tubercular. It was found that the studied individuals are characterized by heterocarpy, which is manifested by qualitative (color, shape, structure of the exocarp) and quantitative characteristics (mass, size of the mericarp, seed, embryo). Mericarps have a smoothed surface or an uneven, lumpy one (Fig. 1. A, B).

The mass of 1000 mericarpies collected in nature from 2.2 to $4.4 \mathrm{~g}$. Mericarps of the 4 th order are the lightest $(2.7 \pm 0.2 \mathrm{~g})$. Mericarpies from umbrellas of the 3rd and 4th order significantly differ in many features. The mericarps are 3.68-5.96 mm long and 2.24-3.62 $\mathrm{mm}$ width. Indicators of fruits from umbrellas of different orders have significant differences, except for the 2 nd and 5th orders. The largest mericarps in umbrellas of the 3rd order are up to $5.97 \mathrm{~mm}(5.61 \pm 0.05 \mathrm{~mm})$ (Fig. 2). The ratio of the width of the mericarp to its length characterizes the shape of the mericarp, its elongation. This indicator varies from 0.48 to 0.75 depending on the order of the umbrella. The mericarps of the umbels of 3-th orders are the most elongated $(0.51 \pm 0.01)$, its of the 4-th orders are the most rounded $(0.61$ \pm 0.01 ). The commissura is wide $3.79 \pm 0.05 \mathrm{~mm}$ long and $1.96 \pm 0.02 \mathrm{~mm}$ width (Fig $1, \mathrm{C}$ ). The spermoderm is thin (unicellular) light brown. The seeds are without endosperm, $3.61 \pm$ $0.04 \mathrm{~mm}$ long. The values of all the studied characters of the mericarp and the seed have significant differences depending on the order of the umbrella on the shoot $\left(t_{\mathrm{st}}=2.10-16.16\right.$, at $\mathrm{p}=0.95)$, except for the mericarps from the umbrellas of the 2 nd and 5 th order $\left(\mathrm{t}_{\mathrm{st}}=0.05-\right.$ 1.21). The embryo is dense, white, differentiated. The length of the embryo (0.54-1.54 
$\mathrm{mm}$ ) depends on the degree of formation and the place of formation on the shoot of the inflorescence, the embryo of mericarp umbrellas of 2-5 order does not differ significantly $\left(\mathrm{t}_{\mathrm{st}}=1.24\right)$, the embryo in the fruits of umbrellas of the 2 nd order is the largest $(1.01 \pm 0.03$ $\mathrm{mm}$ ). Cotyledons are elongated, the length exceeds the width by 1.5-1.7 times (Fig. 1, D).

Allometric features of the embryo characterize its eurthmy, for example, the ratio of the length of the cotyledon to the length of the embryo. This value has no significant differences in embryos of all orders umbrella $\left(\mathrm{t}_{\mathrm{st}}=0.38-1.77\right)$ and varies from 0.42 to 0.68 . On average, cotyledons make up more than half the length of the embryo $(0.55-0.59)$.

Fruits and seeds formed under the conditions of introduction are larger than from natural conditions: weight-1.8-5.7 $\mathrm{g}$, length of the mericarp $-4.17-7.67 \mathrm{~mm}$, the data differ significantly $\left(\mathrm{t}_{\mathrm{st}}=2.50-6.30\right)$. The mericarp measurements are determined by order of the umbrella, the data differ significantly, except mericarps of umbrella of the 2nd and 3rd order (Fig. 2).
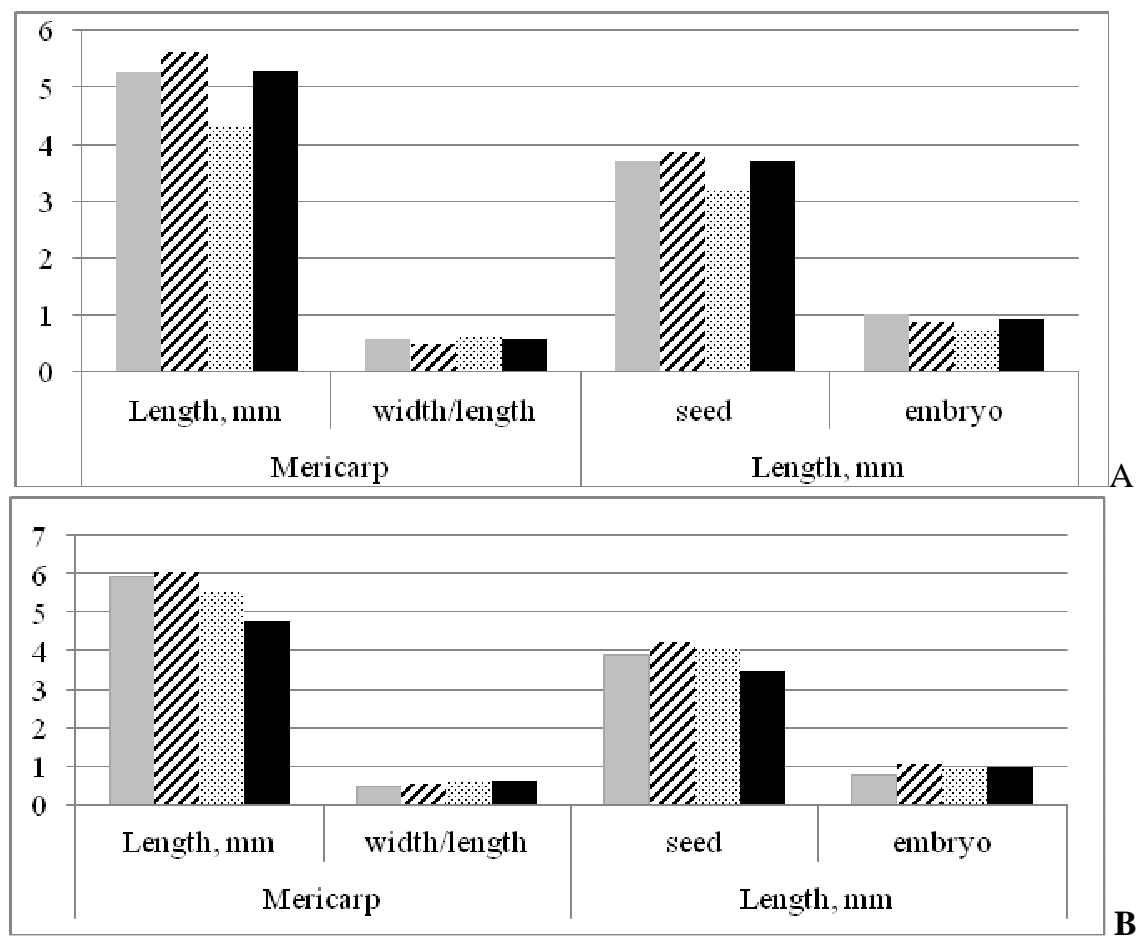

Fig. 2. Biometric characteristics of the mericarp and ta seed of Saposhnikovia divaricate. A -natural

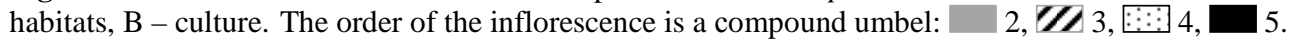

The seed size also significantly differs between the orders, except for the 3rd and 4th order. The largest mericarps were found in umbrellas of the third order. The eurthmy of the embryo in the conditions of culture varies more - from 0.35 to 0.75 . However, it has no significant differences in umbrella embryos of the $2,3,4$ order $\left(t_{\mathrm{st}}=0.75-0.62\right)$ and, on average, cotyledons are half the length of the embryo, as in natural conditions (0.54-0.59).

The length of the cotyledons, the axis of the embryo and the allometric characteristics of the embryo do not have significant differences in fruits collected from nature and in introduction $\left(\mathrm{t}_{\mathrm{st}}=0.8-1.40\right)$. However, the embryo is slightly larger in the fruits collected in culture $\left(\mathrm{t}_{\mathrm{st}}=2.05\right)$. The coefficient of variation of features is from 9 to $22 \%$, regardless of the place of origin of the fruits.

As a result of the conducted studies, it was found that the heterocarpy of fruits in $S$. divaricata is expressed both in qualitative signs (color, surface, etc.) and in quantitative 
signs (the size of the mericarpia, embryo, etc.). The cause of heterocarpy has not been identified, it may be related to the position in the fruit relative to the column, with the position of the fruits on the inflorescence, the position on the generative shoot. It is possible that weather conditions affect the fruits formation on a particular inflorescence. Heterocarpy affects the germination of seeds in Umbelliferae $[5,6]$ and, probably, on their chemical composition. This requires further study.

The studies is carried out according to of the state assignment of the Central Siberian Botanical Garden of the Siberian Branch of the Russian Academy of Sciences state registration no. AAAAA17-117012610051-5 under the project "Assessment of the morphogenetic potential of plant populations of North Asia by experimental methods» and applying materials bioresource scientific scientific collection of the Central Scientific and Biological Center SB RAS, "Collection of living plants in open and closed ground" USU 440534.

\section{References}

1. M Yang., C Wang., W. Wang, J. Xu., J. Wang, C. Zhang, M. Li, Chin. J. Integr. Med., 26 (2020)

2. H. Heuberger, R. Bauer, F. Friedl, G. Heub, J. Hummelsberger, R. Noge, R. Seidenberger, P. Torres-Londono, Planta Med., 76 (2010)

3. M. G. Pimenov, Flora of Siberia, Vol. 10 (Novosibirsk, Nauka, Siberian branch, 1996)

4. G. N. Zaitsev, Mathematical analysis of biological data (Moscow, 1991)

5. S. N. Oparina, Samara Luka: problems of regional and global ecology, 20, 3 (2011)

6. K. Tkachenko, Hortus bot., $15(2020)$ 